\begin{tabular}{c} 
PROBABILITY \\
AND \\
MATHEMATICAL STATISTICS \\
\hline $\begin{array}{c}\text { Vol. 39, Fasc. } 1 \text { (2019), pp. } 61-73 \\
\text { doi:10.19195/0208-4147.39.1.4 }\end{array}$
\end{tabular}

\title{
ON A LIMIT STRUCTURE OF THE GALTON-WATSON BRANCHING PROCESSES WITH REGULARLY VARYING GENERATING FUNCTIONS
}

\author{
BY
}

AZAM A. IMOMOV (TASHKENT)

Dedicated to the fond memory of my Father

\begin{abstract}
We investigate limit properties of discrete time branching processes with application of the theory of regularly varying functions in the sense of Karamata. In the critical situation we suppose that the offspring probability generating function has an infinite second moment but its tail regularly varies. In the noncritical case, the finite moment of type $\mathbb{E}[x \ln x]$ is required. The lemma on the asymptotic representation of the generating function of the process and its differential analogue will underlie our conclusions.
\end{abstract}

2010 AMS Mathematics Subject Classification: Primary: 60J80; Secondary: 60J85.

Key words and phrases: Galton-Watson branching processes, regularly varying functions, transition functions, invariant measures, limit theorems, $Q$-processes.

\section{INTRODUCTION}

Let $\left\{Z_{n}, n \in \mathbb{N}_{0}\right\}$ be the Galton-Watson (G-W) branching process with offspring probability generating function $(\mathrm{GF}) F(s)=\sum_{j \in \mathbb{N}_{0}} p_{j} s^{j}$, where $p_{0}>0$, $p_{1}>0, p_{0}+p_{1}<1, \mathbb{N}_{0}=\{0\} \cup \mathbb{N}$ and $\mathbb{N}=\{1,2, \ldots\}$. The process evolution is characterized by transition probabilities $P_{i j}(n)=\mathbb{P}_{i}\left\{Z_{n}=j\right\}$ for $i \in \mathbb{N}$, where $\mathbb{P}_{i}\{*\}:=\mathbb{P}\left\{* \mid Z_{0}=i\right\}$. Further, where required, we will write $\mathbb{P}\{*\}$ instead of $\mathbb{P}_{1}\{*\}$. In this interpretation, $p_{k}=\mathbb{P}\left\{Z_{1}=k\right\}$ and $m=F^{\prime}(1-)$ is a mean per capita offspring number. The process $\left\{Z_{n}\right\}$ is said to be subcritical, critical and supercritical if $m<1, m=1$ and $m>1$, respectively. A GF $F_{n}(s)=$ $\sum_{j \in \mathbb{N}_{0}} P_{1 j}(n) s^{j}$ is the $n$-fold iteration of $F(s)$, see [2].

Let $\mathcal{H}=\min \left\{n: Z_{n}=0\right\}$ be the time to extinction of the G-W process. The variable $q=\inf \{s \in(0,1]: F(s)=s\}$ is the probability of extinction of our process. By the classical extinction theorem we know that $\mathbb{P}_{i}\{\mathcal{H}<\infty\}=q^{i}$ and that $q=1$ if $m \leqslant 1$, and $q<1$ if $m>1$. 
Our reasoning is involved with elements of the theory of regularly varying functions in sense of Karamata, see [8]. Let us recall that a real-valued, positive and measurable function $\ell(x)$ is said to be slowly varying at $\alpha$ if it belongs to a class

$$
\mathfrak{S}_{\alpha}:=\left\{\ell(x) \in \mathbb{R}_{+}: \lim _{x \rightarrow \alpha} \frac{\ell(\lambda x)}{\ell(x)}=1 \text { for each } \lambda \in \mathbb{R}_{+}\right\}
$$

where and in the sequel $\mathbb{R}_{+}$is the set of positive real numbers. A function $\mathrm{V}(x)$ is said to be regularly varying at $\alpha$ with index of regular variation $\rho \in \mathbb{R}_{+}$if it takes the form $\mathrm{V}(x)=x^{\rho} \ell(x)$, where $\ell(x) \in \mathfrak{S}_{\alpha}$. We denote by $\mathfrak{R}_{\alpha}^{\rho}$ the class of regularly varying functions. It is obvious that $\mathfrak{S}_{\alpha} \equiv \mathfrak{R}_{\alpha}^{0}$, see [11].

The approach to applications of regularly varying functions in the theory of branching processes was first made by Zolotarev [16] in the continuous time case. In the discrete time case, earlier researches belong to Slack [15], [14] and Seneta [13], [12], [110]. The detailed results connected with applications of regularly varying functions in the theory of branching processes can be found in the monographs [I] and [3].

In the noncritical situation, positive numbers

$$
\left\{\nu_{k}:=\lim _{n \rightarrow \infty} \mathbb{P}_{i}\left\{Z_{n}=k \mid n<\mathcal{H}<\infty\right\}\right\}
$$

are an invariant distribution with respect to transition probabilities $\left\{P_{i j}(n)\right\}$. Moreover, the limiting GF $\mathcal{V}(s)=\sum_{j \in \mathbb{N}} \nu_{j} s^{j}$ satisfies the Schröder equation

$$
1-\mathcal{V}(\widehat{F}(s))=\beta \cdot[1-\mathcal{V}(s)]
$$

for all $0 \leqslant s \leqslant 1$, where $\widehat{F}(s)=F(q s) / q$ and $\beta=F^{\prime}(q)$. If in the case $m<1$ we have $\mathbb{E}\left[Z_{1} \ln Z_{1}\right]<\infty$, then $\mathcal{V}^{\prime}(1-)<\infty$ and

$$
\mathbb{P}\{\mathcal{H}>n\} \sim \frac{1}{\mathcal{V}^{\prime}(1-)} \cdot m^{n}
$$

as $n \rightarrow \infty$; see, e.g., [13]].

By the following theorem we see that the regular variation is implicitly present in the equation (1.1) and the asymptote (1.2).

THEOREM S. The following assertions are true:

1. For $m<1$ there exists $\ell(s) \in \mathfrak{S}_{0}$ such that $\ell(s) \downarrow 1 / \mathcal{V}^{\prime}(1-)$ as $s \downarrow 0$ and

$$
\mathbb{P}\{n<\mathcal{H}<\infty\}=\ell\left(m^{n}\right) \cdot m^{n} .
$$

2. In the case $m \neq 1$ the $G F \mathcal{V}(s)$ is a unique solution of (1.1) and

$$
1-\mathcal{V}(1-s) \in \mathfrak{R}_{0}^{1} .
$$


The first part of Theorem S was proved in [3], pp. 398-399; see also [13]. The second part was proved in [U], pp. 60 and 84, separately for the cases $m<1$ and $m>1$.

In the critical case we suppose that the GF $F(s)$ has the following representation:

$\left[\Re_{\nu}\right]$

$$
F(s)=s+(1-s)^{1+\nu} \mathcal{L}\left(\frac{1}{1-s}\right)
$$

for $0 \leqslant s<1$, where $0<\nu<1$ and $\mathcal{L}(t) \in \mathfrak{S}_{\infty}$. By the criticality of our process, the second moment

$$
F^{\prime \prime}(1-)=\lim _{s \uparrow 1} \frac{2(F(s)-s)}{(1-s)^{2}}=\lim _{x \downarrow 0} \frac{2}{x^{1-\nu}} \mathcal{L}\left(\frac{1}{x}\right)=\infty .
$$

Our aim is to generalize the relation (1.3) for $m \neq 1$. For this we prove Lemma 2.1 concerning the asymptote of the function $R_{n}(s)=1-F_{n}(q s) / q$. Then we obtain the asymptotic representations for $\partial R_{n}(s) / \partial s$. These representations entail local limit Theorems 2.1 and 2.2. In Section 3 we investigate the limit structure of the G-W process where all our conclusions will be based on the statements of Lemma 2.2.

\section{BASIC LEMMA AND ITS DIFFERENTIAL ANALOGUE}

We begin with a proof of the following basic lemma of the theory of G-W branching processes (in the literature this name is usually used for a critical case).

LEMMA 2.1. The following assertions are true:

1. Let $m \neq 1$. Then

$$
R_{n}(s)=(1-s) L_{n}(1-s) \cdot \beta^{n},
$$

where $L_{n}(0+)=1$ for all $n \in \mathbb{N}, L_{n}(1)=\ell_{a}\left(\beta^{n}\right)$, with $\ell_{a}(s) \in \mathfrak{S}_{0}$ and $L_{n_{0}}(x)$ $=: L(x) \in \mathfrak{S}_{0}$ for each fixed $n_{0} \in \mathbb{N}$. If $\mathbb{E}\left[Z_{1} \ln Z_{1}\right]<\infty$, then $L_{n}(1) \downarrow 1 / \mu$ as $n \rightarrow \infty$, where $\mu=\mathcal{V}^{\prime}(1-)<\infty$.

2. Let $m=1$. If the condition $\left[\Re_{\nu}\right]$ is satisfied, then

$$
R_{n}(s)=\frac{\mathcal{N}(n+\mathcal{M}(s))}{(\nu n)^{1 / \nu}} \cdot\left[1-\frac{M_{n}(s)}{\nu n}\right],
$$

where the function $\mathcal{N}(x) \in \mathfrak{S}_{\infty}$ satisfies

$$
\mathcal{N}(n) \cdot \mathcal{L}^{1 / \nu}\left(\frac{(\nu n)^{1 / \nu}}{\mathcal{N}(n)}\right) \rightarrow 1 \quad \text { as } n \rightarrow \infty,
$$

and the function $M_{n}(s)$ has the following properties: 
(i) $M_{n}(s)=\mathcal{M}(s)(1+o(1))$ as $n \rightarrow \infty$;

(ii) $\lim _{s \uparrow 1} M_{n}(s)=\nu n$ for each fixed $n \in \mathbb{N}$;

(iii) $M_{n}(0)=0$ for each fixed $n \in \mathbb{N}$.

Pro of. First we will prove the formula (2.1). We use essentially the same method which was applied in the proof of the relation (1.3) in [13]. We rewrite the equation (1.1) as follows:

$$
\vartheta(\phi(s))=\beta \cdot \vartheta(s),
$$

where $\vartheta(s)=1-\mathcal{V}(1-s)$ and $\phi(s):=R_{1}(1-s)$. Putting $\phi_{n}(s):=R_{n}(1-s)$, we arrive at the iteration $\phi_{n+1}(s)=\phi_{n}(\phi(s))$. Hence, from (2.4) it follows that

$$
\vartheta\left(\phi_{n}(s)\right)=\beta^{n} \cdot \vartheta(s) .
$$

Denoting by $a(y)$ the inverse of the function $\vartheta(x)$, we see from (2.5) that

$$
\phi_{n}(s)=a\left(\vartheta(s) \cdot \beta^{n}\right) .
$$

Therefore, we immediately obtain

$$
R_{n}(s)=a\left(\vartheta(1-s) \cdot \beta^{n}\right),
$$

in accordance with our notation.

It has been shown in [13] that $a(s)=s \ell_{a}(s)$, where the function $\ell_{a}(s) \in \mathfrak{S}_{0}$ actually is the same as in equality (1.3). In turn, as shown in Theorem $S, \vartheta(s) \in \mathfrak{R}_{0}^{1}$, so $\vartheta(s)=s \ell_{\vartheta}(s)$, where $\ell_{\vartheta}(s) \in \mathfrak{S}_{0}$. Hence, the equality (2.6) can be written in the form (2.1) where

$$
L_{n}(x)=\ell_{\vartheta}(x) \cdot \ell_{a}\left(x \ell_{\vartheta}(x) \beta^{n}\right) .
$$

Since $\mathcal{V}(0)=0$, we have $\ell_{\vartheta}(1)=1$, and hence $L_{n}(1)=\ell_{a}\left(\beta^{n}\right)$. On the other hand, according to our notation, it can be seen that $a(0)=\vartheta(0)=0$. Therefore, by the property of the inverse functions,

$$
\ell_{a}(0+)=\lim _{x \downarrow 0} \frac{a(x)}{x}=\lim _{x \downarrow 0} \frac{x}{\vartheta(x)}=\frac{1}{\mathcal{V}^{\prime}(1-)} .
$$

As noticed above, the GF $\mathcal{V}(s)$ satisfies the Schröder equation (1.1), where $\widehat{F}(s)=$ $F(q s) / q$ is the GF of the subcritical branching process. In this case, as mentioned above, $\mathcal{V}(s)$ has the finite mean $\mu=\mathcal{V}^{\prime}(1-)$ if and only if $\mathbb{E}\left[Z_{1} \ln Z_{1}\right]<\infty$. Hence $L_{n}(1)=\ell_{a}\left(\beta^{n}\right) \downarrow 1 / \mu$. Similarly, it is easy to see that

$$
\ell_{\vartheta}(0+)=\lim _{x \downarrow 0} \frac{\vartheta(x)}{x}=\mu,
$$

which implies that $L_{n}(0+)=1$. 
Finally, by the property of slowly varying functions we see that the function $L_{n_{0}}(x)=: L(x) \in \mathfrak{S}_{0}$ at any fixed $n_{0} \in \mathbb{N}$. Thus, the proof of the first part of Lemma 2.1 is completed.

Let us prove now the second part of the lemma. By analogy with the continuous time Markov branching process (see [6], p. 111), it follows that the function

$$
\mathcal{M}(s)=\int_{0}^{s} \frac{d x}{F(x)-x}
$$

is a power series in $s \in[0,1)$ with nonnegative coefficients, $\mathcal{M}(s)=\sum_{j \in \mathbb{N}} \mu_{j} s^{j}$, and $\left\{\mu_{j}, j \in \mathbb{N}\right\}$ is an invariant measure for the $\mathrm{G}-\mathrm{W}$ process. Using the condition $\left[\Re_{\nu}\right]$ in the last integrand, we write the equality

$$
\mathcal{M}(s)=\int_{1}^{1 /(1-s)} u^{\nu-1} \mathcal{L}^{-1}(u) d u .
$$

Now we recall the following property of slowly varying functions. If the function $\ell(x) \in \mathfrak{S}_{\infty}$ is locally bounded in $[A ;+\infty)$ for some $A \in \mathbb{R}_{+}$, then

$$
\int_{A}^{x} u^{\lambda} \ell(u) d u \sim \frac{1}{\lambda+1} x^{\lambda+1} \ell(x) \quad \text { as } x \rightarrow \infty
$$

for $\lambda>-1$; see [3], p. 26. Since the upper bound of the integral on the right-hand side of (2.7) goes to infinity as $s \uparrow 1$, we can use this property and get the following asymptotic formula:

$$
\mathcal{M}(s)=\frac{1+o(1)}{\nu(1-s)^{\nu} \mathcal{L}(1 /(1-s))} \quad \text { as } s \uparrow 1 .
$$

Denoting by $G(x)$ the inverse of the function $\mathcal{M}(1-s)$ and using (2.8), we obtain

$$
G(x)=\frac{\mathcal{N}(x)}{(\nu x)^{1 / \nu}},
$$

where $\mathcal{N}(x) \in \mathfrak{S}_{\infty}$ satisfies (2.3); see also [9].

On the other hand, it is known that the following Abel equation holds true (if normalized so that $\left.\mathcal{M}\left(p_{0}\right)=1\right)$ :

$$
\mathcal{M}\left(F_{n}(s)\right)=\mathcal{M}(s)+n \quad \text { for all } n \in \mathbb{N} ;
$$

see, e.g., [2], p. 68.

Combining the equations (2.9) and (2.10), we obtain

$$
R_{n}(s)=\frac{\mathcal{N}(n+\mathcal{M}(s))}{(\nu n+\nu \mathcal{M}(s))^{1 / \nu}}=\frac{\mathcal{N}(n+\mathcal{M}(s))}{(\nu n)^{1 / \nu}} \cdot \frac{1}{(1+\mathcal{M}(s) / n)^{1 / \nu}} .
$$


By the convergence of the integral (2.7), the second factor in this equality tends to one as $n \rightarrow \infty$. Therefore, the equality can be written in the form

$$
\frac{1}{(1+\mathcal{M}(s) / n)^{1 / \nu}}=1-\frac{M_{n}(s)}{\nu n},
$$

where $M_{n}(s)$ is some function. Observe now some local and asymptotic properties of the function $M_{n}(s)$. It follows from equality (2.11) that

$$
1-\frac{M_{n}(s)}{\nu n} \sim 1-\frac{\mathcal{M}(s)}{\nu n} \quad \text { as } n \rightarrow \infty .
$$

Therefore, $M_{n}(s) \rightarrow \mathcal{M}(s)$ as $n \rightarrow \infty$. In turn, since $\mathcal{M}(1-)=\infty$, it follows from (2.11) that $\lim _{s \uparrow 1} M_{n}(s)=\nu n$ for each fixed $n \in \mathbb{N}$. Finally, $M_{n}(0)=0$ because $\mathcal{M}(0)=0$.

The lemma is proved completely.

Note that the first part of Lemma 2.1 contains formula (1.3). In fact, setting $s=0$, from (2.1) we infer that for $m \neq 1$

$$
\mathbb{P}\{n<\mathcal{H}<\infty\}=q-F_{n}(0)=q \cdot \ell\left(\beta^{n}\right) \cdot \beta^{n} .
$$

The second part is a discrete analogue of a similar result from [5]. In particular, putting $s=0$ in (2.2), we obtain

$$
\mathbb{P}\{\mathcal{H}>n\}=R_{n}(0)=\frac{\mathcal{N}(n)}{(\nu n)^{1 / \nu}},
$$

an extension probability of the critical G-W process.

LEMMA 2.2. The following assertions are true:

1. Let $m \neq 1$. Then

$$
\frac{\partial R_{n}(s)}{\partial s}=-\frac{R_{n}(s)}{1-s}-(1-s) L_{n}^{\prime}(1-s) \cdot \beta^{n} \quad \text { as } n \rightarrow \infty .
$$

2. Let $m=1$. If the condition $\left[\Re_{\nu}\right]$ is satisfied, then

$$
\frac{\partial R_{n}(s)}{\partial s}=-\left(\frac{R_{n}(s)}{1-s}\right)^{1+\nu} \frac{\mathcal{L}\left(1 / R_{n}(s)\right)}{\mathcal{L}(1 /(1-s))} .
$$

Pro of. As to the first part of the lemma, we previously noticed that due to (2.1) we have

$$
\beta^{-n} R_{n}(1-s)=s L_{n}(s) \in \mathfrak{R}_{0}^{1} \quad \text { with respect to } s,
$$

for any fixed $n \in \mathbb{N}$. And we see that the function $r_{n}(s)=s L_{n}(s)$ has a derivative $r_{n}^{\prime}(s)=L_{n}(s)+s L_{n}^{\prime}(s)$. Hence, we obtain the formula (2.12). 
To prove the second part, we differentiate the equation (2.10):

$$
F_{n}^{\prime}(s)=\frac{\mathcal{M}^{\prime}(s)}{\mathcal{M}^{\prime}\left(F_{n}(s)\right)} .
$$

In turn, differentiating the formula (2.7) gives

$$
\mathcal{M}^{\prime}(s)=\frac{1}{(1-s)^{1+\nu} \mathcal{L}(1 /(1-s))} .
$$

Thus the equality (2.13) follows from (2.14) and (2.15).

The lemma is proved.

Using Lemma 2.2, we directly obtain $P_{11}(n)=F_{n}^{\prime}(0)$ the probability of return of the process to the initial state $Z_{0}=1$ in time $n$. In the case of $m \neq 1$, it follows from the form of $L_{n}(x)=\ell_{\vartheta}(x) \cdot \ell_{a}\left(x \ell_{\vartheta}(x) \beta^{n}\right)$ that

$$
L_{n}^{\prime}(s)=\frac{\ell_{\vartheta}^{\prime}(s)}{\ell_{\vartheta}(s)} \cdot L_{n}(s)+\mathcal{O}\left(\beta^{n}\right) \quad \text { as } n \rightarrow \infty .
$$

Note that $\ell_{\vartheta}^{\prime}(1)=\mathcal{V}^{\prime}(0)-1$ because $\ell_{\vartheta}(s)=\vartheta(s) / s$. Hence, setting $s=0$ in (2.12), we obtain the following local limit theorem.

THEOREM 2.1. Let $m \neq 1$. Then the following relation holds true:

$$
\beta^{-n} \cdot P_{11}(n)=\mathcal{V}^{\prime}(0) \cdot \ell_{a}\left(\beta^{n}\right)+\mathcal{O}\left(\beta^{n}\right) \text { as } n \rightarrow \infty .
$$

If $\mathbb{E}\left[Z_{1} \ln Z_{1}\right]<\infty$, then $\ell_{a}\left(\beta^{n}\right) \downarrow 1 / \mu$ as $n \rightarrow \infty$, where $\mu=\mathcal{V}^{\prime}(1-)<\infty$.

Similarly, from (2.13) we get the following theorem.

THEOREM 2.2. Let $m=1$. If the condition $\left[\Re_{\nu}\right]$ holds, then

$$
(\nu n)^{1+1 / \nu} \cdot P_{11}(n) \sim \frac{\mathcal{N}(n)}{p_{0}} \quad \text { as } n \rightarrow \infty,
$$

where the function $\mathcal{N}(n) \in \mathfrak{S}_{\infty}$ satisfies the relation (2.3).

At the end of the section we define the stochastic process $\left\{\widetilde{Z}_{n}\right\}$ with transition matrix $\widetilde{P}_{i j}(n)=\mathbb{P}_{i}\left\{Z_{n}=j \mid n<\mathcal{H}<\infty\right\}$. As noticed at the beginning, in the noncritical case the limiting distribution $\left\{\nu_{j}:=\lim _{n \rightarrow \infty} \widetilde{P}_{i j}(n)\right\}$ does not depend on $i \in \mathbb{N}$. So $\left\{\widetilde{Z}(n), n \in \mathbb{N}_{0}\right\}$ is the ergodic Markov chain. Thus, in the case $m \neq 1$, there exists an ergodic chain concerned with the process $\left\{Z_{n}\right\}$ such that its transition probabilities are

$$
\widetilde{P}_{i j}(n)=\frac{P_{i j}(n) q^{j}}{\sum_{k \in \mathbb{N}} P_{i k}(n) q^{k}} .
$$

Let $\mathcal{V}_{n}^{(i)}(s)=\sum_{j \in \mathbb{N}} \widetilde{P}_{i j}(n) s^{j}$. The following two theorems are corollaries of Lemma 2.1 and the fact that $\mathcal{V}_{n}^{(i)}(s) \sim 1-R_{n}(s) / R_{n}(0)$ as $n \rightarrow \infty$. 
THEOREM 2.3. If $m \neq 1$, then the limiting $G F \mathcal{V}(s)=\lim _{n \rightarrow \infty} \mathcal{V}_{n}^{(i)}(s)$ converges for $0 \leqslant s \leqslant 1$ with $\mathcal{V}(0)=0$ and $\mathcal{V}(1)=1$. Furthermore,

$$
\frac{1-\mathcal{V}_{n}^{(i)}(s)}{1-s} \rightarrow \ell(1-s) \quad \text { as } n \rightarrow \infty,
$$

where $\ell(x) \in \mathfrak{S}_{0} ;$ moreover, if $\mathbb{E}\left[Z_{1} \ln Z_{1}\right]<\infty$, then $\ell(0+)=\mu$ and $\ell(1)=1$.

THEOREM 2.4. Let $m=1$. If the condition $\left[\Re_{\nu}\right]$ holds, then

$$
\nu n \cdot \mathcal{V}_{n}^{(i)}(s)=\mathcal{M}(s)(1+o(1)) \quad \text { as } n \rightarrow \infty,
$$

where $\mathcal{M}(s)=\sum_{j \in \mathbb{N}} \mu_{j} s^{j}$ satisfies the Abel functional equation (2.10). For probabilities $\widetilde{P}_{i j}(n)$ the following relation holds:

$$
\nu n \cdot \widetilde{P}_{i j}(n) \rightarrow \mu_{j} \quad \text { as } n \rightarrow \infty,
$$

and $\sum_{j \in \mathbb{N}} \mu_{j}=\infty$.

\section{FURTHER APPLICATIONS OF LEMMA 2.2}

The second part of Lemma 2.2 might be used in the proof of the following theorem whose analogue for the case $F(1-x)-(1-x) \in \mathfrak{R}_{0}^{1+\nu}$ was established by Slack [14]. In our case we give the proof based on the ideas of the SteinTikhomirov method, see [7].

THEOREM 3.1. Let $m=1$ and $q_{n}:=\mathbb{P}\{\mathcal{H}>n\}$. If the condition $\left[\Re_{\nu}\right]$ holds, then

$$
G_{n}^{(i)}(x):=\mathbb{P}_{i}\left\{q_{n} Z_{n}<x \mid \mathcal{H}>n\right\}
$$

weakly converges to a limit law $G(x)$ having the Laplace transform

$$
\Psi(\theta):=\int_{\mathbb{R}_{+}} e^{-\theta s} d G(s)=1-\frac{\theta}{\left(1+\theta^{\nu}\right)^{1 / \nu}}, \quad \theta \in \mathbb{R}_{+} .
$$

Proof. Since $1-F_{n}^{i}(s) \sim i R_{n}(s)$ as $n \rightarrow \infty$, it suffices to consider the Laplace transform for $i=1$ :

$$
\Psi_{n}(\theta):=\int_{\mathbb{R}_{+}} e^{-\theta s} d G_{n}^{(1)}(s)=1-\frac{R_{n}\left(\theta_{n}\right)}{q_{n}},
$$

where $\theta_{n}=\exp \left\{-\theta q_{n}\right\}$. Differentiating (3.1), we have

$$
\Psi_{n}^{\prime}(\theta)=\theta_{n} R_{n}^{\prime}\left(\theta_{n}\right)
$$


By the definition of the slowly varying functions, it follows from the second part of Lemma 2.1 and formula (2.13) that

$$
R_{n}^{\prime}\left(\theta_{n}\right) \sim-\left(\frac{R_{n}\left(\theta_{n}\right)}{1-\theta_{n}}\right)^{1+\nu} \quad \text { as } n \rightarrow \infty .
$$

From (3.1)-(3.3) it follows that

$$
\Psi_{n}^{\prime}(\theta) \sim-\left(\frac{1-\Psi_{n}(\theta)}{\theta}\right)^{1+\nu} \quad \text { as } n \rightarrow \infty .
$$

It is easy to check that $\Psi(\theta)$ is a solution of the equation

$$
\Psi^{\prime}(\theta)=-\left(\frac{1-\Psi(\theta)}{\theta}\right)^{1+\nu} .
$$

Hence, according to the Stein-Tikhomirov method, the equation (3.4) is equivalent to the convergence $\Psi_{n}(\theta) \rightarrow \Psi(\theta)$. Thus, the theorem is proved.

Now consider a conditional distribution

$$
\mathbb{P}_{i}^{\mathcal{H}(n+k)}\{*\}:=\mathbb{P}_{i}\{* \mid n+k<\mathcal{H}<\infty\} .
$$

In [2], p. 56, it was shown that the probability measure

$$
\mathcal{Q}_{i j}(n):=\lim _{k \rightarrow \infty} \mathbb{P}_{i}^{\mathcal{H}(n+k)}\left\{Z_{n}=j\right\}=\frac{j q^{j-i}}{i \beta^{n}} P_{i j}(n)
$$

defines the Markov chain $\left\{W_{n}, n \in \mathbb{N}_{0}\right\}$ with state space $\mathcal{E} \subset \mathbb{N}$, called the $Q$ process. By the definition,

$$
\mathcal{Q}_{i j}(n)=\mathbb{P}_{i}\left\{Z_{n}=j \mid \mathcal{H}=\infty\right\},
$$

and the $Q$-process can be interpreted as the $\mathrm{G}-\mathrm{W}$ process with non-degenerate trajectory in the distant future. In terms of the generating functions, the equality (3.5) can be written as follows:

$$
Y_{n}^{(i)}(s):=\sum_{j \in \mathcal{E}} \mathcal{Q}_{i j}(n) s^{j}=\left[\frac{F_{n}(q s)}{q}\right]^{i-1} Y_{n}(s),
$$

where the GF $Y_{n}(s):=Y_{n}^{(1)}(s)=\mathbb{E}\left[s^{W_{n}} \mid W_{0}=1\right]$ has the form

$$
Y_{n}(s)=-s \frac{R_{n}^{\prime}(s)}{\beta^{n}} \quad \text { for all } n \in \mathbb{N},
$$

and, as before, $R_{n}(s)=1-F_{n}(q s) / q$. 
From (3.6) and (3.7) we get the following functional equation:

$$
Y_{n+1}^{(i)}(s)=\frac{Y(s)}{\widehat{F}(s)} Y_{n}^{(i)}(\widehat{F}(s)),
$$

where $Y(s):=Y_{1}(s)$.

THEOREM 3.2. Let $\beta<1$. If $\mathbb{E}\left[\ln W_{1}\right]<\infty$, then there exists a limit function $\pi(s):=\lim _{n \rightarrow \infty} Y_{n}^{(i)}(s)$ for $0 \leqslant s<1$ and

$$
\pi(s)=\frac{1}{\mu} s \mathcal{V}^{\prime}(s),
$$

where $\mathcal{V}(s)$ is the $G F$ of the invariant measure of the $G-W$ process $\left\{Z_{n}\right\}$ satisfying the Schröder equation (1.1) and $\mu=\mathcal{V}^{\prime}(1-)$. Moreover, the $G F \pi(s)=$ $\sum_{j \in \mathcal{E}} \pi_{j} s^{j}$ generates an invariant distribution $\left\{\pi_{j}, j \in \mathcal{E}\right\}$ for the $Q$-process.

Pr o o f. Since $R_{n}(s) \rightarrow 0$ uniformly for $0 \leqslant s<1$, it follows from (3.6) and (3.7) that $\mathcal{Q}_{i j}(n) / \mathcal{Q}_{1 j}(n) \rightarrow 1$ as an infinite growth of the number of generations, so it suffices to consider the case $i=1$. From (2.1) and (2.12) we obtain

$$
R_{n}^{\prime}\left(s=-\left[L_{n}(1-s)+(1-s) L_{n}^{\prime}(1-s)\right] \cdot \beta^{n},\right.
$$

where $L_{n}(x)=\ell_{\vartheta}(x) \cdot \ell_{a}\left(x \ell_{\vartheta}(x) \beta^{n}\right)$. Using this equality in formula (3.7) and taking into account a derivative of the function $L_{n}(x)$, we have

$$
Y_{n}(s)=s L_{n}(1-s) \cdot\left[1+(1-s) \frac{\ell_{\vartheta}^{\prime}(1-s)}{\ell_{\vartheta}(1-s)}+\mathcal{O}\left(\beta^{n}\right)\right]
$$

as $n \rightarrow \infty$. By the definition of the $Q$-process, the condition $\mathbb{E}\left[\ln W_{1}\right]<\infty$ is equivalent to the assumption $\mathbb{E}\left[Z_{1} \ln Z_{1}\right]<\infty$, which in turn is enough for the existence of $\mu=\mathcal{V}^{\prime}(1-)$. In the proof of the first part of Lemma 2.1 we have shown that $\ell_{a}(0+)=1 / \mu$. In the relation (3.10), we use the form of the function $L_{n}(x)$ and properties of the function $\ell_{\vartheta}(s)=\vartheta(s) / s \in \mathfrak{S}_{0}$, and consequently we have

$$
\begin{aligned}
\pi(s) & =\frac{1}{\mu} s \ell_{\vartheta}(1-s)\left[1+(1-s) \frac{\ell_{\vartheta}^{\prime}(1-s)}{\ell_{\vartheta}(1-s)}\right] \\
& =\frac{1}{\mu} s \ell_{\vartheta}(1-s)+\frac{1}{\mu} s\left[\mathcal{V}^{\prime}(s)-\ell_{\vartheta}(1-s)\right],
\end{aligned}
$$

which implies the formula (3.9).

The equation (3.8) gives

$$
\pi(s)=\frac{Y(s)}{\widehat{F}(s)} \cdot \pi(\widehat{F}(s))
$$


which is equivalent to the invariance property $\pi_{j}=\sum_{i \in \mathcal{E}} \pi_{i} \mathcal{Q}_{i j}(1)$ of the sets of numbers $\left\{\pi_{j}, j \in \mathcal{E}\right\}$ generated by $\pi(s)=\sum_{j \in \mathcal{E}} \pi_{j} s^{j}$. It is exactly a probability distribution. In fact, it follows from (3.9) that $\pi(1)=\sum_{j \in \mathcal{E}} \pi_{j}=1$. Thus, the theorem is proved.

THEOREM 3.3. Let $\beta=1$. If the condition $\left[\Re_{\nu}\right]$ holds, then

$$
\frac{(\nu n)^{1+1 / \nu}}{\mathcal{N}(n)} \cdot Y_{n}^{(i)}(s)=\mathcal{U}(s)(1+o(1)) \quad \text { as } n \rightarrow \infty
$$

for all $0 \leqslant s<1$. The limiting $G F \mathcal{U}(s)=\sum_{j \in \mathcal{E}} v_{j} s^{j}$ takes the form

$$
\mathcal{U}(s)=\frac{s}{(1-s)^{1+\nu} \mathcal{L}(1 /(1-s))} .
$$

The set of coefficients $\left\{v_{j}, j \in \mathcal{E}\right\}$ is an invariant measure for the Q-process. Moreover,

$$
\sum_{j=1}^{n} v_{j} \sim \frac{1}{\Gamma(2+\nu)} n^{1+\nu} \mathcal{L}_{v}(n) \quad \text { as } n \rightarrow \infty,
$$

where $\Gamma(*)$ is the Euler's gamma function and $\mathcal{L}_{v}(n) \cdot \mathcal{L}(n) \rightarrow 1$ as $n \rightarrow \infty$.

Pro of. As in Theorem 3.2 we consider the case $i=1$ only. Combining the relations (2.2), (2.13) and (3.7) yields

$$
Y_{n}(s)=s\left(\frac{\mathcal{N}(n+\mathcal{M}(s))}{(\nu n)^{1 / \nu}(1-s)}\right)^{1+\nu} \frac{\mathcal{L}\left(1 / R_{n}(s)\right)}{\mathcal{L}(1 /(1-s))}\left[1-\frac{M_{n}(s)}{\nu n}\right]^{1+\nu} .
$$

Using (2.3) and the property of the slowly varying functions, we have

$$
Y_{n}(s)=\frac{\mathcal{N}(n)}{(\nu n)^{1+1 / \nu}} \cdot \frac{s}{(1-s)^{1+\nu} \mathcal{L}(1 /(1-s))}(1+o(1))
$$

as $n \rightarrow \infty$, where we have used the properties of the function $M_{n}(s)$. Multiplying the both sides of (3.14) by $(\nu n)^{1+1 / \nu} / \mathcal{N}(n)$, we obtain the convergence (3.11) with limiting function of the form (3.12).

Taking the limit as $n \rightarrow \infty$ in (3.8) gives

$$
\mathcal{U}(s)=\frac{Y(s)}{F(s)} \cdot \mathcal{U}(F(s)),
$$

which implies an invariance property of the numbers $\left\{v_{j}, j \in \mathcal{E}\right\}$. Finally, (3.13) is a direct consequence of (3.12), according to the Hardy-Littlewood Tauberian theorem for the GF; see [4], pp. 513-514. Thus, the theorem is proved. 
Since $\lim _{s \downarrow 0}\left[Y_{n}^{(i)}(s) / s\right]=\mathcal{Q}_{i 1}(n)$, the following local properties of the $Q$ process follow from the last two theorems.

Corollary 3.1. Let $\beta<1$. If $\mathbb{E}\left[\ln W_{1}\right]<\infty$, then

$$
\mathcal{Q}_{i 1}(n) \rightarrow \frac{\mathcal{V}^{\prime}(0)}{\mu} \text { as } n \rightarrow \infty \text {. }
$$

COROLlaRY 3.2. Let $\beta=1$. If the condition $\left[\Re_{\nu}\right]$ holds, then

$$
(\nu n)^{1+1 / \nu} \cdot Q_{i 1}(n) \sim \frac{\mathcal{N}(n)}{p_{0}} \quad \text { as } n \rightarrow \infty,
$$

where the function $\mathcal{N}(n) \in \mathfrak{S}_{\infty}$ satisfies the relation (2.3).

Thus, two types of the $Q$-process depending on the value of the parameter $\beta$ can be seen. In the case $\beta=1$, the trajectory of the $Q$-process goes to infinity with probability one. Hence, the Markov chain $\left\{W_{n}\right\}$ is transient if $\beta=1$. It is positive recurrent if $\beta<1$.

Finally, continuing the reasoning in the proof of Theorem 3.1, we state the following result whose analogue for the case $F(1-s)-(1-s) \in \mathfrak{R}_{0}^{1+\nu}$ has been established in [9].

THEOREM 3.4. Let $\beta=1$. If the condition $\left[\Re_{\nu}\right]$ holds, then $\mathbb{P}\left\{q_{n} W_{n}<x\right\}$ weakly converges to a limiting distribution law with Laplace transform

$$
\frac{1}{\left(1+\theta^{\nu}\right)^{1+1 / \nu}}
$$

Pr o o f. According to (3.7), the Laplace transform of $\mathbb{P}\left\{q_{n} W_{n}<x\right\}$ is $\varphi_{n}(\theta)$ $=-\Psi_{n}^{\prime}(\theta)$, where $\Psi_{n}^{\prime}(\theta)$ is defined in (3.2). Since, as it has been established in Theorem 3.1,

$$
\Psi_{n}(\theta) \rightarrow 1-\frac{\theta}{\left(1+\theta^{\nu}\right)^{1 / \nu}} \quad \text { as } n \rightarrow \infty,
$$

it follows from (3.4) that $\varphi_{n}(\theta)$ converges as $n \rightarrow \infty$ to the limit expression mentioned in the theorem.

Acknowledgments. The author thanks the anonymous referee for the careful reading of the manuscript and for his kindly comments which contributed to improving the paper.

\section{REFERENCES}

[1] S. Asmussen and H. Hering, Branching Processes, Birkhäuser, Boston 1983.

[2] K. B. Athreya and P. E. Ney, Branching Processes, Springer, New York 1972. 
[3] N. H. Bingham, C. M. Goldie, and J. L. Teugels, Regular Variation, Cambridge University Press, Cambridge 1987.

[4] W. Feller, An Introduction to Probability Theory and Its Applications, Vol. 2, Mir, Moscow 1967 (Russian edition).

[5] Sh. K. Formanov and Zh. B. Azimov, Markov branching processes with regularly varying generating function and immigration of a special form, Theory Probab. Math. Statist. 65 (2002), pp. 181-188.

[6] T. E. Harris, The Theory of Branching Processes, Springer, Berlin 1963.

[7] A. A. Imomov, A differential analog of the main lemma of the theory of Markov branching processes and its applications, Ukrainian Math. J. 57 (2) (2005), pp. 307-315.

[8] J. Karamata, Sur un mode de croissance régulière: Théorèmes fondamentaux, Bull. Soc. Math. France 61 (1933), pp. 55-62.

[9] A. G. Pakes, Some new limit theorems for the critical branching process allowing immigration, Stochastic Process. Appl. 3 (1975), pp. 175-185.

[10] E. Seneta, On the invariant measures for simple branching processes, J. Appl. Probab. 8 (1971), pp. 43-51.

[11] E. Seneta, Regularly Varying Functions, Springer, Berlin 1972.

[12] E. Seneta, A Tauberian theorem of E. Landau and W. Feller, Ann. Probab. 1 (1973), pp. 1057-1058.

[13] E. Seneta, Regularly varying functions in the theory of simple branching processes, Adv. in Appl. Probab. 6 (1974), pp. 408-420.

[14] R. S. Slack, A branching process with mean one and possible infinite variance, Z. Wahrsch. Verw. Gebiete 9 (1968), pp. 139-145.

[15] R. S. Slack, Further notes on branching processes with mean 1, Z. Wahrsch. Verw. Gebiete 25 (1972), pp. 31-38.

[16] V. M. Zolotarev, More exact statements of several theorems in the theory of branching processes, Theory Probab. Appl. 2 (1957), pp. 245-253.

Azam A. Imomov

State Testing Center under the Cabinet of Ministers

of the Republic of Uzbekistan

12, Bogishamol St.

100202, Tashkent, Uzbekistan

Karshi State University, 17, Kuchabag St.

180100, Karshi City, Uzbekistan

E-mail:imomov_azam@mail.ru

Received on 6.3.2017;

revised version on 4.8 .2017 